\title{
Predicting Heart Diseases from Large Scale IoT Data Using a Map-Reduce Paradigm
}

https://doi.org/10.1515/comp-2020-0204

Received Jan 06, 2020; accepted Mar 03, 2020

\begin{abstract}
Over the last few years, the huge amount of data represented a major obstacle to data analysis. Big data implies that the volume of data undergoes a faster progress than computational speeds, thereby demanding a larger data storage capacity. The Internet of Things (IoT) is a main source of data that is closely related to big data, as the former extends to a variety of fields such as healthcare, entertainment, and disaster control. Despite the different advantages associated with the composition of Big Data analytics and IoT, there are a number of complex difficulties and issues involved that need to be resolved and managed to ensure an accurate data analysis. Some of these solutions include the utilization of map-reduce techniques, processing, and large data scale, particularly for the relatively less time that this method requires to process large data from the Internet of Things. Machine learning algorithms of this kind are often implemented in the healthcare sector. Medical facilities need to be advanced so that more appropriate decisions can be made in terms of patient diagnosis and treatment options. In this work, two datasets have been used: the first set, used in the prediction of heart diseases, obtained an accuracy rate of 84.5 for RF and 83 for J48, whereas the second dataset is related to weather stations (automated sensors) and obtained accuracy rates of 88.5 and 86.5 for RF and J48, respectively.
\end{abstract}

Keywords: Internet of Things (IoT), Big Data, map-reduce, HDF, Random Forest (RF) algorithm

\section{Introduction}

Nowadays, internet applications tend to have a vital role in our daily life, as these can be used in social media commu-

\footnotetext{
*Corresponding Author: Mehdi Ebady Manaa: University of Babylon, College of IT, Department of Information Networks; Email: meh_man12@yahoo.com; it.mehdi.ebady@itnet.uobabylon.edu.iq Faris Mohammad Abd: University of Babylon, College of IT, Department of Information Networks; Email: farismoh421@gmail.com
}

nication, advertising, recommendation systems, health care, e-mails, e-learning and many others. The virtual space provides a suitable platform to put in force different applications so as to simplify difficult techniques in diverse domain names. This is caused by the spread of various sensor styles and the significant increase in the number of computers, consequently resulting in the creation of more data, called the "Internet of Things (IoT)" [2]. Significant quantities of internet data are also generated from other sources, such as videos, texts, and audio fragments created by users. It has been noticed that the IoT has benefited people in different fields including healthcare, particularly in terms of preventing medication errors [3]. For example, there are around 50 billion sensor-based devices that depend on the internet and it is expected that there will be over 6.58 devices per individual by 2020 [4].

The use of IoT seems to contribute to the enhancement of the living conditions for many people. A lot of advantages related to connecting sensor data or networking between sensors are used in a wide range of applications, including environmental control [5], disaster control [6], health care [7], and smart system of cities [8]. It is also of use to scientific societies [9], as the IoT provides many items for accurate research of data through its access to several sensors, thereby supplying various forms of datasets. On the other hand, by utilizing these "Things", we participate in the (big) data flow. IoT can thus be defined as a means of data collecting that provides a stream of information in vast quantities from billions of Internetlinked objects [10]

\subsection{Big Data Definition and Characteristics}

Big data is defined as a substantial volume of data (both structured and unstructured), with no significance of representing the actual amount of data [11].

In 2000, a telescope in New Mexico gathered more data on earth's beginnings than had ever been accumulated in the history of astronomy. After a decade, its archive included about 140 terabytes of data. Since 2016, there is another big survey telescope in Chile, which has been predicted to gather the same amount of data every 5 days [11].

๑ Open Access. (c) 2020 F. M. Abd and M. E. Manaa, published by De Gruyter. 
More recently, Facebook, a social media platform, stores over 500 terabytes of data every day whereas the search engine manages 20 petabytes of data on a daily basis [11] According to these examples, we are able to quantify the amount of data the world processes and how fast the data volume is developing. In 2003, a total of five exabytes of data had been formed throughout the year by individuals, whereas nowadays this same amount of data is formed within two days only. In 2012, the quantity of data in the digital world has reached over 2.72 zettabytes and has doubled annually since, being extended by a further eight zettabytes by 2015 [12]. This rapid increase in data quantity, as well as the fact that it becomes more complicated, makes it hard to use data processing techniques.

Big data has three major criteria, described as 3Vs (volume, velocity and variety). Figure 1 shows these main criteria.

A. Volume: This criterion represents the most immediate challenge to traditional IT structures. Many corporations already have massive amounts of archived data in the shape of logs, but do not own the ability to process that data [13]. The advantage gained from the capacity to process massive amounts of information is the major attraction of huge data analytics.

B. Velocity: Velocity refers to the increase in speed at which the data is created. Increasing the velocity of data creation leads to the fast processing, saving, and analyzing of data [13].

C. Variety: The last aspect refers to the different data types such as text, audio, pictures, movies, and many additional unstructured or semi-structured forms of data [14]. An example would be the communication through social media platforms on a daily basis: 900 million photos are shared and watched on Facebook, five hundred million tweets are shared on Twitter, 0.4 million hours of video are seen on YouTube, and 3 billion searches are uploaded to Google [13]. These numbers could be described as a massive data explosion. Huge amounts of data contribute to such an explosion, merely by admitting the coming flow of data and processing it simultaneously so that no bottlenecks are produced.

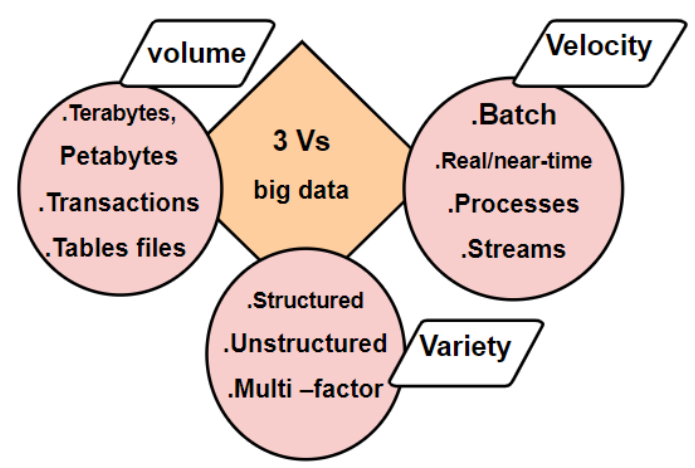

Figure 1: Big data characteristics

\subsection{Map-Reduce Programming Model}

Map-Reduce runs on several groups of commodity hardware for computing depending on java. It is a typical software program layout, run for the processing and management of gigantic information sets [15]. The MR model consists of two significant functions: the Map (M) function that procedures a key-value combination for supplying a group of couples of intermediate key-value, and the Reduce (R) function which completely integrates intermediate values related with intermediate key. Programs composed in a beneficial model are automatically in parallel and provide substantive trade goods mechanisms that monitor the division of input files, create the program's run across branches of mechanisms, and understand both the machine fails and the aimed inter-machine communication. This allowed programmers to create parallel and divided arrangements, thereby making the resources of a great number of distributed systems available. MapReduce uses the technique of (GFS) as a main storage layer to analyze the data of input and then save the data of output [16].

Google file systems are blocks-based and deliver file system that helps error-tolerance by means of information used in division and repetition. Apache Hadoop is an external frame of application of the Map-Reduce algorithm [17].

The Hadoop architecture includes two layers. The first one is a data storage called Hadoop DFS (HDFS), whereas the other is an information process called (Hadoop MapReduce Framework). Hadoop DFS is a block-structured filing system controlled by only one master node as Google's GFS [18]. 


\subsection{Hadoop Distributed File System (HDFS)}

The Hadoop Distributed File System (HDFS) is the programming style that analyzes data by processing resources in the cluster. Every node within a Hadoop cluster takes a model of the master or slave. Master nodes always include the name node of the job tracker [19]. Slave nodes often include both the Data Node and the Task Tracker. The task in task tracker could be one or more iteration in every cluster. Figure 2 shows the Hadoop architecture.

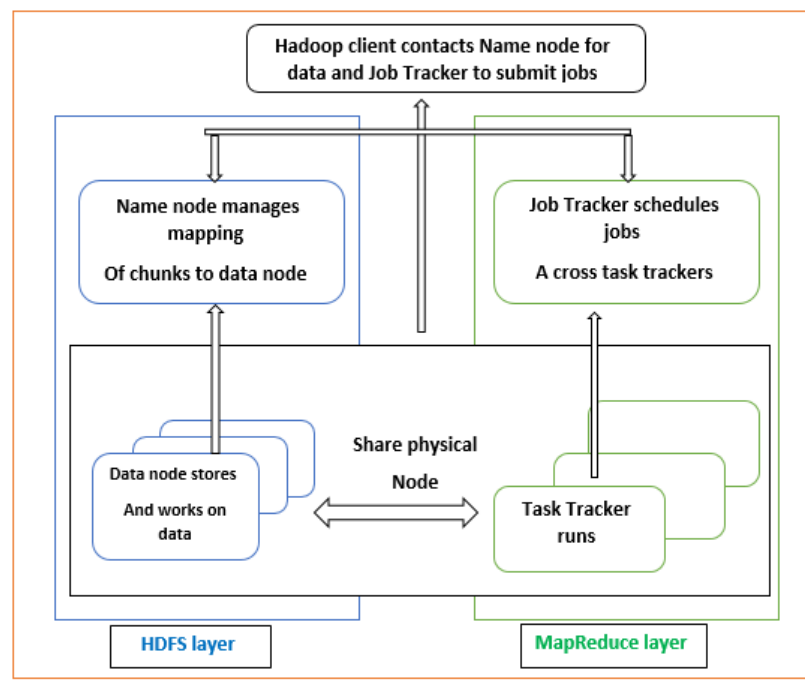

Figure 2: Hadoop architecture

\section{Related Works}

The authors in [20] presented a solution to cope with the big data that has recently increased, using the traditional Relational Database Management System (RDBMS). However, this system became ineffective as it consumes a large time scale to perform a huge data analysis. Therefore, Hadoop is now utilized to overcome the restrictions of traditional RDBMS through providing simple techniques which might in turn result in effective data package while being less time consuming. Furthermore, it becomes an analytical tool through the use of MapReduce, Hive and Pig.

In [21], the MapReduce provides an effective route to manage a large quantity of data. Through their research, the authors assessed and verified the IoTDeM, stating that they were able of successfully reducing the total performance time of MapReduce techniques and applications, with a relatively lower error average of less than ten percent.
In [22], it is stated that cardiovascular and heart diseases are found to be among the major causes of mortality and morbidity, thus forming the necessity of being able to predict the occurrence of such diseases. An early detection could prevent and/or manage such, often fatal, diseases. In this matter, data mining techniques such as support vector machine SVM, KNN, decision tree DT, Naive Bayes NB and trained neural network NN, are used and a MapReduce technique is performed.

In [23], the authors explained that the creation of data in web searches is massive, and it continues to develop rapidly in volume within short time intervals. This data should be treated and taken in consideration, for which they suggested the use of an (MR) algorithm that would arrange and isolate the various difficulties in web, eventually improving the outcomes in the arrival to web sites.

\section{The Problem Statement}

The huge quantity of data that complicates its processing within a suitable period of time represents a major problem of large-scale data. In addition to the longer time needed to extract the information, there are problems in the actual analysis of the data that lead to incomplete or inaccurate results, which in turn impede the building of a robust data mining model. One of the solutions that have been suggested is the use of MapReduce, as the reduce task has the ability to decrease the huge amount of data by applying a certain method of counting them. Other contributions involve the reduction of average time consumption, as well as obtaining more precise results. In addition, it should provide a massive storage of data and unlimited processing power [24].

\section{The Proposed System}

In this research, health data has been used, through which many common heart diseases are investigated. This data was taken from the United Kingdom, December 2-5 (2014). It is an extract from the most common diseases that were processed by Hadoop2x. Figure 3 shows the main steps of the proposed system. 


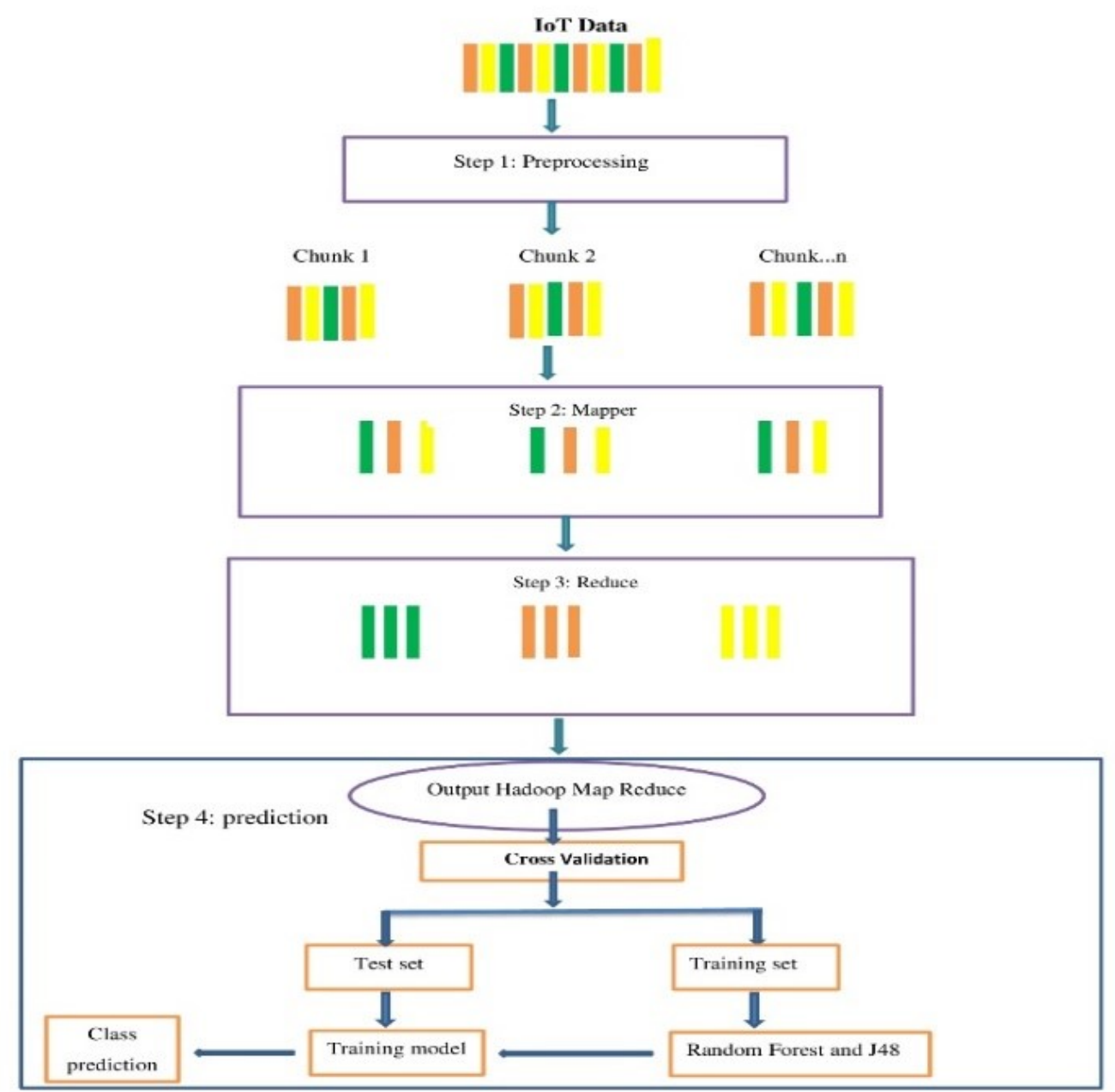

Figure 3: The main steps of the proposed system

\subsection{Materials and methods}
A) Input Files and Preprocessing
B) Splitting
C) Mapping
D) Shuffling and Reducing
E) Prediction IoT disease data
F) Evaluation

Step 1 involves the preprocessing of the large IoT data, which is then input into Hadoop MapReduce. In Step 2, the data is divided into several blocks by MapReduce. The function of MapReduce obtained two phases, a map phase followed by a reduce phase. Step 3 is for the map phase to process every input instance and create modern key-value couple. Step 4 requires the Reducer phase to receive the group of intermediate key-value couples produced by the mappers as the input, after which it carries out a reducer task on each one of them to produce the output. The output of reducer is the last output that is isolated in HDFS. Step 5 continues the process with the Hadoop output for heart diseases that can be predicted using the machinelearning model. The Random forest model was utilized in the exception phase. Step 6 represents the evaluation phase, which involves the inputting group of data (test data) on the model. The performance of MapReduce is evaluated using 20 million records, so the data is processed in a seconds. Algorithm 1-3 show the main steps of the mapreduce and driver respectively.

\subsection{Map Algorithm}

The Map task is the first phase in the MapReduce model. It receives input functions (considered as datasets) and separates them into minimal sub-functions. After that, any 
computation that may be required is performed on every sub-function in parallel.

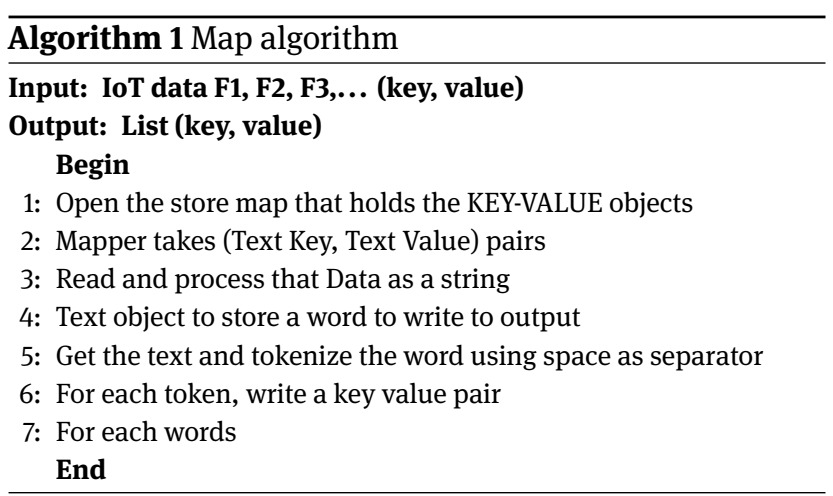

by passing various parameters using mapper and reducer classes, input, output, and others.

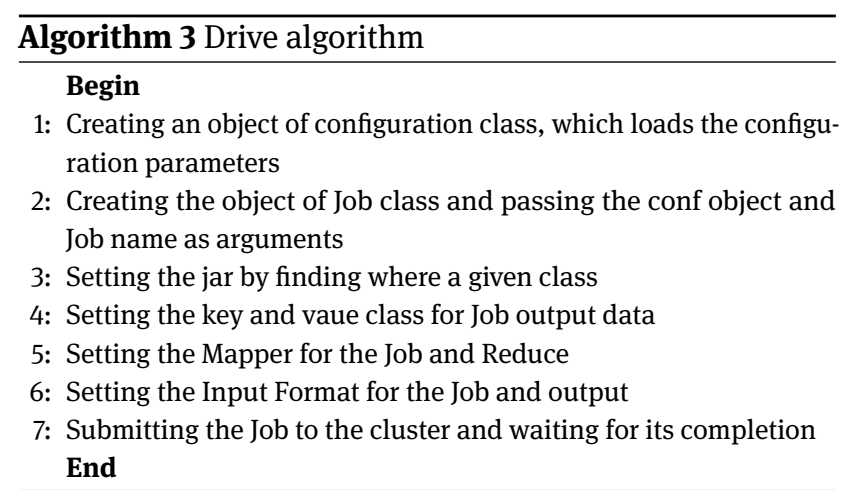

\subsection{Reduce Algorithm}

The reduce algorithm receives a listing of <Key, List<Value $>>$ sorted couples from Shuffle task and implements the reduce step. The output of the Mapper class is utilized like an input via the Reducer class, which seeks matching couples and reduces them. This phase is the integration of the Shuffle phase and the Reduce phase. The Reducer's task is processing the data that is delivered from the mapper.

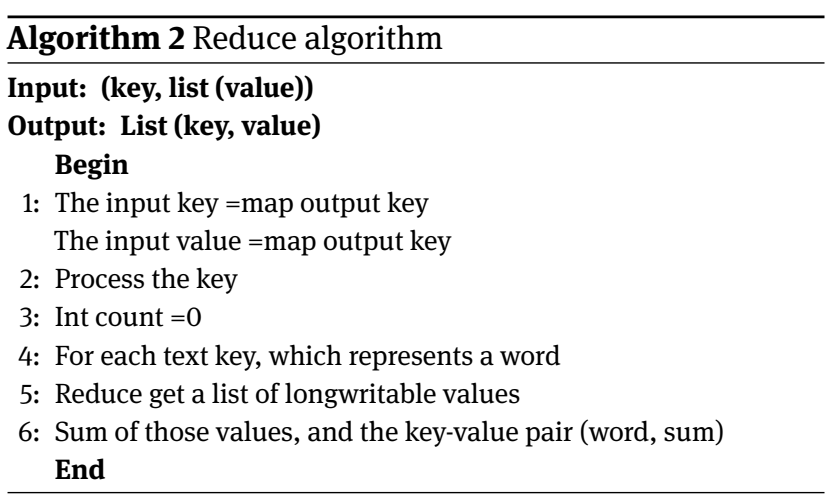

\subsection{Random Forest}

This is a brand word for a staff of decision trees, in which there are clusters of decision trees (hence the term Forest). To categorize a modern subject dependent on characters; every tree determines a categorization and the tree is considered as votes for that category. The random forest chooses the categorization that obtains the high votes (through the trees in this forest) [25].

\subsection{Decision tree (j48)}

This model produces the basics for the exception of the target variable. The J48 classier is utilized to determine the target value of a new symbol that depends on various attributes of the data. The inner nodes of the decision tree indicate the various attributes; but the divisions between nodes point to the potential values of those at tributes, whereas the final nodes establishes the categorization of the target [26].

\subsection{Naïve Bayes}

The Naive Bayes model is a simple probability algorithm, which works by calculating the frequency and aggregations of values in a certain data set. The algorithm uses Bayes's theory and supposes that the whole parameter is independent considering the value of the class variable [27]. the required set of parameters. The code associated with the driver class is referred to as driver code. A driver class is responsible for the execution of the MapReduce functions 


\subsection{REPTree}

The REPTree algorithm is a rapid decision tree learner that positions the acquiring of information as the splitting criterion, and clips it using the reduced error clipping way. The Reduced Error Clipping method provides an easier and more accurate categorization tree [28].

\subsection{Performance Evaluation}

- Kappa statistic: It is a measurement of how closely the instances categorized via the machine learning classifier correspond or match the data labeled as actual data. The change corrected represents a measure of correspondence between the categorizations and the correct categories [29].

- Mean absolute error: It is the collection of the definitive errors (or deviations) quantity utilized to measure how close forecasts or exceptions are to the definitive result.

- Root mean squared error (RMSE): It is the square root of the mean of the squares of the values. It squares the errors before the mean is calculated, and the RMSE gives a relatively high weight to big errors.

- Relative Absolute Error: It is the complete absolute error made relative to what the error would have been if the exception had easily been the mean of the real values.

- Root relative squared error: is the complete squared error made relative to what the error would have been if the exception had been the mean of the absolute value. Like the root mean-squared error, the square root of the relative squared error provides similar dimensions as the expected value.

\subsection{Apache Hadoop Computers Characteristics}

This table presents the specifications of each compute node, IP Address and the count of nodes that represents master and slave in the Apache Hadoop multi node clus-

Table 1: The specification of the Hadoop compute Node

\begin{tabular}{ccccc}
\hline Node IP & Behavior & CPU & RAM & OS type \\
\hline 192.168 .0 .100 & Master & Core i5 & $3.8 \mathrm{~GB}$ & 64-bit \\
192.168 .0 .101 & Slave 1 & Core i5 & $3.8 \mathrm{~GB}$ & 64-bit \\
192.168 .0 .102 & Slave 2 & Core i3 & $3.8 \mathrm{~GB}$ & 64-bit \\
\hline
\end{tabular}

ter. Table 1 shows the characteristics of each node in the Hadoop.

\section{Data Set Description}

Throughout the present work, two datasets have been used as follows:

\subsection{The Heart Disease Data}

The data used in this study is the United Kingdom Heart disease data set available at [30]. The volume of dataset used is about one million records and it has 24 raw attributes. Six column attributes are used in the study of 6160 records (see Table 2).

Table 2: The description of the heart disease data

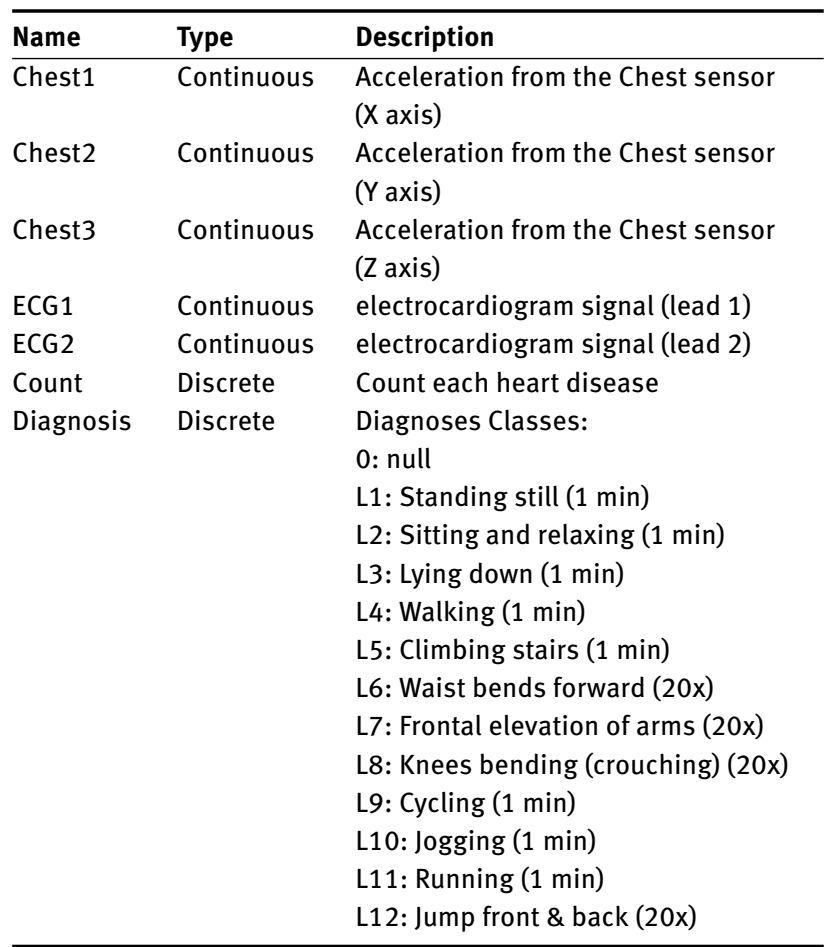

The dataset that is used involves ten volunteers, for whom the motions of the body and vital signs have been recorded when doing twelve daily activities. All datasets are merged and combined together in a single dataset in order to be used and processed via the MapReduce. For the readings, the sensors were used, as these were put on the person's chest, left ankle and right wrist. Utilizing them allows us to record movement, called acceleration, via any 
part of the body, as well as the average of turn and magnetic field orientation. The measurements are utilized in order to efficiently record all possible motion, for example, during major monitoring of the heart. Heart diseases represent a major cause of mortality and morbidity, as around $25 \%$ of the fatal cases worldwide (for both genders) are due to conditions of the heart. In this research, various arrhythmias (sinus ventricular tachycardia or bradycardia), atrial fibrillation, ectopic beats were investigated, after which the consequences of excessive exercise on the readings of ECG were determined. The frequency of the sample that is used is fifty hertz, which is enough for recording physical daily activities. Everyone was registered through video in one minute. This dataset is recorded in order to generalize well-known physical activities, to show the different body parts involved in every movement (for example, the bending of knees and elevation of arms), the density of the movement (for example sitting and relaxing), as well as the speed of the motion, as in standing and running.

The motions were recorded under room temperature of the lab investigator, with the assumption that the volunteer is doing their best in these activities. Machine learning is used, and then the prediction of dataset is made through using the algorithm of random forest. In random forest, the physical daily activities are classified into 13 classes (null, standing still, sitting and relaxing, etc.). The cross validation feature is used to classify the data randomly.

\subsection{The second dataset: Weather Stations (Automated Sensors)}

The data used in this study is the weather data at beaches along Chicago's Lake Michigan lakefront, created in 2019 and obtained from the data.gov site. Sensors are used to capture the determined measurements every hour during the summer. The volume of the dataset used is around 97.000 instances and it has 19 raw attributes. Eight columns with attributes are used in the study of 4000 instances. The cross-validation feature is used to classify the data randomly.

\section{Results and Discussion}

Four computers were used with the simple specification in the installation process; Java is installed first, then Hadoop 2x is used because it is faster than Hadoop version 1 and had the characteristic of Yarn. Cluster resource administration and processing are carried out using vari- ous processing tools. Also, Hadoop 2x has a higher scalability limit i.e. up to 10.000 nodes per cluster, in contrast to Hadoop 1x that has lower scale nodes, less than 4000 nodes per cluster.

Table 3 explains the empirical outcomes. Several trials have been performed to estimate the achievement and benefits of various categorization models, so as to identify patients with heart disease.

Table 3: The results of Prediction diseases with using the Apache Hadoop for the Heart Disease

\begin{tabular}{ccc}
\hline \multirow{2}{*}{ Evaluation Criteria } & \multicolumn{2}{c}{ Prediction } \\
\cline { 2 - 3 } & Random Forest & J48 \\
\hline Correctly Classified Instances & 3692 & 3627 \\
Incorrectly Classified Instances & 675 & 740 \\
Accuracy (\%) & $84.5 \%$ & $83 \%$ \\
\hline
\end{tabular}

The data presented in Table 3 indicates that the $\mathrm{RF}$ classifier is more accurate and precise than $\mathrm{j} 48$, as the number of correctly classified instances is higher than $\mathrm{j} 48$ and the number of incorrectly classified instances is less than $\mathrm{j}$ 48. The rate of properly categorized cases is usually referred to as accuracy. Random Forest is a set of decision trees that represents effective modeling and tends to be much stronger than $\mathrm{J} 48$ which has one decision tree. The implementation of the learning modalities is highly dependent on the training data. Figure 4 shows the comparison between Random Forest and J48 in terms of numbers, correct and error instances.

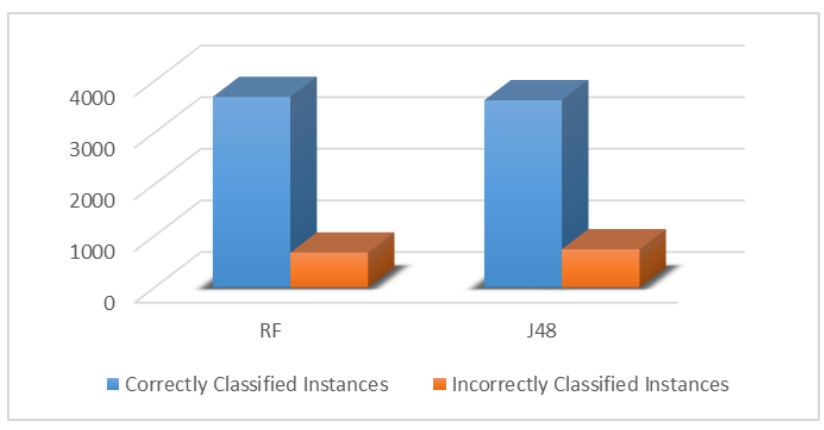

Figure 4: The comparison between Random Forest and J48 for the Heart Disease

The Kappa statistic refers to unlimited error, and the root mean squared error is in numeric value only. The relative absolute error and root relative squared error are show in percentages for the purpose of reference and evaluation. The results of the simulation are shown in Table 4. 
Table 4: The statistical evaluation of RF and J48 in Map-Reduce for the Heart Disease

\begin{tabular}{ccc}
\hline \multirow{2}{*}{ Evaluation Criteria } & \multicolumn{2}{c}{ Prediction } \\
\cline { 2 - 3 } & Random Forest & $\mathbf{J 4 8}$ \\
\hline Kappa Statistic & 0.78 & 0.74 \\
Mean Absolute Error (MAE) & 0.43 & 0.04 \\
Root Mean Squared Error (RMSE) & 0.14 & 0.17 \\
Relative Absolute Error (RAE) & $31.10 \%$ & $31.08 \%$ \\
Root relative squared error & $55.09 \%$ & $65.75 \%$ \\
\hline
\end{tabular}

Table 4 shows the performance of the Kappa Statistic applied to the RF and J48 classifier technique on the dataset, with a difference in the size of the training and testing sets. Kappa Statistic in Random Forest is 0.79 in spite of using MapReduce, but Random Forest keeps its position on top. Random Forest Classifier has minimum errors when Hadoop is used in terms of Mean Absolute Error (MAE), Root Mean Square Error (RMSE), Relative Absolute Error (RAE) and Root Relative Squared Error (RRSE) when compared with other methods. Table 5 explains the empirical outcome after performing many trials to estimate the achievement and benefits of various categorization models, so as to predict weather stations using Random forest and $\mathrm{J} 48$ algorithm.

Table 5 illustrates how RF remains on the top in the second dataset by achieving higher accuracy than $\mathrm{j} 48$. It is shown that the number of correctly classified instances of $R F$ is higher than $j 48$, and the number of incorrectly classified instances of RF is less than for $j 48$.

Table 5: The results of predicting weather stations using the Apache Hadoop

\begin{tabular}{ccc}
\hline \multirow{2}{*}{ Evaluation Criteria } & \multicolumn{2}{c}{ Prediction } \\
\cline { 2 - 3 } & Random Forest & $\mathbf{J 4 8}$ \\
\hline Correctly Classified Instances & 3378 & 3190 \\
Incorrectly Classified Instances & 438 & 626 \\
Accuracy (\%) & $88.5 \%$ & $83.5 \%$ \\
\hline
\end{tabular}

\section{Discussion of Results}

In practice, the classifiers of Random Forest and $\mathrm{J} 48$ achieved better results in the prediction of heart diseases and weather forecasts than the other classifiers such as Naive Bayes and REPTree.

Most of the classifiers, including RF, j48, Naive Bayes and others, have been applied on both datasets (Heart Dis- ease Data, Weather Stations (Automated Sensors)), and it was found that RF achieved a more accurate prediction rate than all other classifiers. Table 6 shows the difference between the algorithms.

Table 6: The difference between Classification algorithms

\begin{tabular}{lcc}
\hline Algorithm name & $\begin{array}{c}\text { First dataset: } \\
\text { Heart Disease Data } \\
\text { (accuracy (\%)) }\end{array}$ & $\begin{array}{c}\text { Second dataset: } \\
\text { Weather Stations } \\
\text { (accuracy (\%)) }\end{array}$ \\
\hline Random Forest & $84.5 \%$ & $88.5 \%$ \\
J48 & $83 \%$ & $83.3 \%$ \\
Naive Bayes & $81 \%$ & $73.3 \%$ \\
RepTree & $79.4 \%$ & $82.8 \%$ \\
\hline
\end{tabular}

Table 7 draws a comparison of periods between the huge data of IoT heart disease that is used before and after implementation of Hadoop and machine learning classifiers. The prediction is performed and the results are recorded. The results after using MapReduce are better and faster than before using this method.

Table 7: The results of time before and after Hadoop for the loT Heart Disease Data

\begin{tabular}{lcll}
\hline $\begin{array}{l}\text { Number } \\
\text { records }\end{array}$ & Number of files & \multicolumn{2}{c}{ Analysis Time (sec) } \\
\cline { 3 - 4 } $\begin{array}{c}\text { before } \\
\text { Hadoop }\end{array}$ & & $\begin{array}{l}\text { Before using } \\
\text { Hadoop apache }\end{array}$ & $\begin{array}{l}\text { After using } \\
\text { Hadoop apache }\end{array}$ \\
\hline $\mathbf{6 1 6 0}$ & 1 & $29 \mathrm{sec}$ & $18 \mathrm{sec}$ \\
$\mathbf{2 4 6 4 0}$ & 4 & $1 \mathrm{~min} 56 \mathrm{sec}$ & $1 \mathrm{~min} 12 \mathrm{sec}$ \\
$\mathbf{4 3 1 2 0}$ & 7 & $3 \mathrm{~min} 23 \mathrm{sec}$ & $2 \mathrm{~min} 6 \mathrm{sec}$ \\
$\mathbf{6 1 6 0 0}$ & 10 & $4 \mathrm{~min} 50 \mathrm{sec}$ & $\min$ \\
\hline
\end{tabular}

\section{Conclusion}

There is a substantial number of classification and clustering algorithms that process large data IoT heart disease data, yet there is still a need for an effective and inexpensive solution to process big IoT data, as well as to reduce the time of processing. In this paper, we have used a large data of IoT that has been processed by Hadoop. We have suggested a MapReduce programming paradigm for analyzing data that produces IoT of huge data. With the assistance of the Hadoop Technique, we investigated the MapReduce algorithm in terms of both time and performance. The experimental results show that the MapReduce algorithm in big-scale IoT data processing is practi- 
cal and efficient. Thus, the suggested algorithm is suitable for huge scale IoT data processing. We proposed a model to predict diseases of the heart through using data mining methods. Classifiers such as RF and j48 were utilized in order to diagnose heart disease patients. The monitoring records indicated that the RF performance is more precise when compared to the other categorization ways. The top model, which depends on the data of patient, is the RF Categorization, which has an accuracy of $80.56 \%$.

\section{References}

[1] S. Kumar Basak, M. Wotto, and P. Bélanger, "E-learning, Mlearning and D-learning: Conceptual definition and comparative analysis," E-Learning Digit. Media, vol. 15, no. 4, pp. 191-216, 2018.

[2] J. Qi, P. Yang, G. Min, O. Amft, F. Dong, and L. Xu, "Advanced internet of things for personalised healthcare systems: A survey," Pervasive Mob. Comput., vol. 41, pp. 132-149, 2017.

[3] M. Marjani et al., "Big loT data analytics: architecture, opportunities, and open research challenges," IEEE Access, vol. 5, pp. 5247-5261, 2017.

[4] Suzuki, Larissa R. "Smart cities IoT: Enablers and technology road map." In Smart City Networks, pp. 167-190. Springer, Cham, 2017

[5] J. M. Talavera et al., "Review of loT applications in agro-industrial and environmental fields," Comput. Electron. Agric., vol. 142, pp. 283-297, 2017.

[6] A. Sinha, P. Kumar, N. P. Rana, R. Islam, and Y. K. Dwivedi, “Impact of internet of things (IoT) in disaster management: a tasktechnology fit perspective," Ann. Oper. Res., vol. 283, no. 1-2, pp. 759-794, 2019.

[7] M. Elhoseny, G. Ramírez-González, O. M. Abu-Elnasr, S. A. Shawkat, N. Arunkumar, and A. Farouk, "Secure medical data transmission model for loT-based healthcare systems," leee Access, vol. 6, pp. 20596-20608, 2018.

[8] Y. Mehmood, F. Ahmad, I. Yaqoob, A. Adnane, M. Imran, and S. Guizani, “Internet-of-things-based smart cities: Recent advances and challenges," IEEE Commun. Mag., vol. 55, no. 9, pp. 16-24, 2017.

[9] Musleh, Ahmed S., Mahdi Debouza, and Mohamed Farook. "Design and implementation of smart plug: An Internet of Things (IoT) approach." In 2017 International Conference on Electrical and Computing Technologies and Applications (ICECTA), pp. 1-4. IEEE, 2017.

[10] A. Munir, P. Kansakar, and S. U. Khan, "IFCloT: Integrated Fog Cloud IoT: A novel architectural paradigm for the future Internet of Things.," IEEE Consum. Electron. Mag., vol. 6, no. 3, pp. 74-82, 2017.

[11] T. Economist, "Data, data everywhere: A special report on managing information," Econ., 2010.

[12] Sagiroglu, Seref, and Duygu Sinanc. "Big data: A review." In 2013 international conference on collaboration technologies and systems (CTS), pp. 42-47. IEEE, 2013.

[13] Ishwarappa and J. Anuradha, "A brief introduction on big data 5 Vs characteristics and hadoop technology," Procedia Comput.
Sci., vol. 48, no. C, pp. 319-324, 2015.

[14] Y. Dandawate, "Big Data: Challenges and Opportunities, volume 11 of Infosys Labs Briefings. Infosys Labs.” 2013.

[15] S. Regha and M. Manimekalai, "HADOOP VS BIG DATA," 2015.

[16] Z. Lu, N. Wang, J. Wu, and M. Qiu, "IoTDeM: An IoT Big Dataoriented MapReduce performance prediction extended model in multiple edge clouds,” J. Parallel Distrib. Comput., vol. 118, pp. 316-327, 2018.

[17] T. White, “Hadoop: The Definitive Guide, Yahoo.” Press, 2010.

[18] Z. Khanam and S. Agarwal, "Map-reduce implementations: survey and performance comparison," Int. J. Comput. Sci. Inf. Technol.(IJCSIT), vol. 7, no. 4, 2015.

[19] T. S. Buda, H. A. A. A. Salama, L. Xu, P. J. O'sullivan, C. Thorpe, and L. Almeida, "Data replication in a distributed file system." Google Patents, 26-Sep-2019.

[20] P. Vishruti and M. D. Ingle, "Disease Analytics in Healthcare System Using Hadoop,” Int. J. Mod. Trends Sci. Technol., vol. 4, 2018

[21] Z. Lu, N. Wang, J. Wu, and M. Qiu, "IoTDeM: An IoT Big Dataoriented MapReduce performance prediction extended model in multiple edge clouds," J. Parallel Distrib. Comput., vol. 118, pp. 316-327, 2018.

[22] T. Nagamani, S. Logeswari, and B. Gomathy, "Heart Disease Prediction using Data Mining with Mapreduce Algorithm," no. 3, pp. 137-140, 2019.

[23] T. Mylsami and B. L. Shivakumar, "Role of Map reduce Algorithm to Improve," vol. 5, no. 11, pp. 47-51, 2017.

[24] A. Kamilaris, A. Kartakoullis, and F. X. Prenafeta-Boldú, "A review on the practice of big data analysis in agriculture," Comput. Electron. Agric., vol. 143, no. September, pp. 23-37, 2017, doi: 10.1016/j.compag.2017.

[25] T. Mahboob, S. Irfan, and A. Karamat, "A machine learning approach for student assessment in E-learning using Quinlan's C4. 5, Naive Bayes and Random Forest algorithms," in 2016 19th International Multi-Topic Conference (INMIC), pp. 1-8. IEEE, 2016.

[26] T. M. Lakshmi, A. Martin, R. M. Begum, and V. P. Venkatesan, "An analysis on performance of decision tree algorithms using student's qualitative data," Int. J. Mod. Educ. Comput. Sci., vol. 5, no. 5, p. 18, 2013.

[27] F. Paquin, J. Rivnay, A. Salleo, N. Stingelin, and C. Silva, "Multiphase semicrystalline microstructures drive exciton dissociation in neat plastic semiconductors," J. Mater. Chem. C, vol. 3, pp. 10715-10722, 2015.

[28] M. Belouch, S. El Hadaj, and M. Idhammad, "A two-stage classifier approach using reptree algorithm for network intrusion detection," Int. J. Adv. Comput. Sci. Appl., vol. 8, no. 6, pp. 389394, 2017.

[29] S. Gupta and N. Verma, "Comparative Analysis of classification Algorithms using WEKA tool," Int. J. Sci. Eng. Res., vol. 2018, 2014.

[30] Banos, Oresti, Rafael Garcia, Juan A. Holgado-Terriza, Miguel Damas, Hector Pomares, Ignacio Rojas, Alejandro Saez, and Claudia Villalonga. "mHealthDroid: a novel framework for agile development of mobile health applications." In International workshop on ambient assisted living, pp. 91-98. Springer, Cham, 2014. 